\title{
Mecanismos de protección y prevención de las complicaciones de la obesidad en los adolescentes
}

\author{
Mechanisms of protection and prevention of obesity complications in adolescents \\ Imelda C. Zavaleta-Chi ${ }^{a}$, Estefanía Olivares-Palacios ${ }^{b}$, \\ Sandra Olvera-Sosa ${ }^{c}$, Azucena E. Jiménez-Corona ${ }^{d}$.
}

\begin{abstract}
:
Different surveys and assessment instruments of the pediatric population in Mexico have reported that this part of the population is in the first places of overweight and obesity in the country. This fact is extremely alarming due to the negative prognosis of comorbidity and morbidity of the possible pathologies that this population can develop and present in the short and medium term. Therefore, this review is focused on publicizing the causal mechanisms and the origin of the pathologies caused by a metabolic imbalance caused by overweight and obesity. Likewise, the protective mechanisms that endogenous and exogenous antioxidants exert for the prevention of obesity and / or to reverse possible damages caused by the prooxidant agents present in a cellular microenvironment of oxidative stress are described. In this context, it is essential to carry out preventive measures to avoid and / or reverse the risk and the development of some or some chronicodegenerative diseases, through the intake of antioxidants from the diet and the absolute benefit of carrying out a constant routine of aerobic exercise that promotes good health.
\end{abstract}

\section{Keywords:}

Adolescents, obesity, prevention, antioxidants, oxidative stress.

\section{Resumen:}

Diferentes encuestas e instrumentos de valoración de la población pediátrica en México han reportado, que esta parte de la población se encuentra en los primeros lugares de sobrepeso y obesidad a nivel mundial. Este hecho, es sumamente alarmante debido al pronóstico negativo de comorbilidad y morbilidad de las posibles patologías que pueden desarrollar y presentar esta población a corto y mediano plazo. Por lo tanto, esta revisión está enfocada en dar a conocer los mecanismos causales y el origen de las patologías originadas por un desequilibrio metabólico provocado por el sobrepeso y la obesidad. Con la finalidad de dar soluciones y mejorar el estado de salud de esta población y de la población en general, se describen los mecanismos protectores que ejercen los antioxidantes endógenos y exógenos para la prevención de la obesidad y/o revertir posibles daños causados por los agentes prooxidantes presentes en un microambiente celular de estrés oxidativo. En este contexto, es indispensable llevar a cabo medidas preventivas para evitar y/o revertir el riesgo y el desarrollo de alguna o algunas enfermedades crónicodegenerativas, mediante la ingesta de antioxidantes provenientes de la dieta y el absoluto beneficio de llevar a cabo una rutina constante de ejercicio aeróbico que promueve el buen estado de salud.

\section{Palabras Clave:}

Adolescentes, obesidad, prevención, antioxidantes, estrés oxidativo.

\footnotetext{
a Autor de Correspondencia, Profesora Lic. Enfermería, Escuela Superior de Huejutla, Universidad Autónoma del Estado de Hidalgo. Email: imelda_zavaleta11032@uaeh.edu.mx

${ }^{\text {b}}$ Estudiante de Enfermería, Escuela Superior de Huejutla, Universidad Autónoma del Estado de Hidalgo. Email: ol397073@uaeh.edu.mx

c Profesora Lic. Médico Cirujano, Escuela Superior de Huejutla, Universidad Autónoma del Estado de Hidalgo. Email: sandra_olvera@uaeh.edu.mx.

d Profesora-Investigadora de Tiempo Completo, Escuela Superior de Huejutla, Universidad Autónoma del Estado de Hidalgo. ORCID: 0000-0002-8987506X, Email: azucena_jimenez@uaeh.edu.mx
} 


\section{Introducción}

La obesidad es una acumulación anormal o excesiva de grasa que pone en riesgo la salud ${ }_{1}$ y se ha convertido en un problema de salud pública grave que debe atenderse de forma urgente. Generalmente esta enfermedad trae consigo comorbilidades como hipertensión arterial, dislipidemias, enfermedad pulmonar obstructiva crónica (EPOC), cardiopatías, cáncer, estrés y ansiedad en la población general ${ }_{2}$. Actualmente la población pediátrica, primordialmente niños en edad escolar y adolescentes, consumen en mayor cantidad productos industrializados con alto contenido de grasas y azúcares, desplazando así la ingesta de frutas y verduras; al mismo tiempo, se ha observado una disminución en la actividad física en los niños e inadecuados hábitos alimenticios, los cuales están influenciados por la publicidad, ya sea de forma disimulada o intencional y que finalmente tiende a ser un aspecto negativo para la salud. Estas costumbres se han encargado de posicionar a la obesidad infantil como uno de los más serios retos de la salud pública del siglo XXI.

\section{Obesidad en la adolescencia en México}

En el pasado esporádicamente se veían casos de obesidad en niños y adolescentes, hoy en día, la presencia de obesidad en México es común, lo que resulta alarmante es el número de casos que se presentan en este tipo de población.

La UNIFEC hace mención de que México ocupa el primer lugar mundial en obesidad infantil ${ }_{3,4}$. Al ingreso a la primaria (seis años de edad), la prevalencia promedio de sobrepeso y obesidad es del $24.3 \%$; sin embargo, cuando están por concluir la primaria (12 años de edad), la prevalencia se incrementa al $32.5 \%$, lo que refleja 12.2 puntos porcentuales de aumento 5 . Cuando analizamos estas cifras, es cuando podemos dimensionar la situación de malnutrición en la que se encuentra la población infantil.

La encuesta nacional de nutrición (ENSANUT) indicó un incremento de sobrepeso en la población mexicana, específicamente niños preescolares y adolescentes, relacionado por el deficiente estado de salud del individuo que viene marcado desde el vientre materno hasta llegar a la edad adulta ${ }_{6}$.

De acuerdo a los resultados de ENSANUT en los cuales se compara la prevalencia de sobrepeso y obesidad en edades pediátricas, en los preescolares de género femenino se reportó una disminución de la prevalencia de obesidad de $3.9 \%$, siendo de $9.7 \%$ en el 2012 y de $5.8 \%$ en el 2016 , de la misma manera el género masculino registró una disminución de $3.4 \%$, de $9.9 \%$ a $6.5 \%$ en el año 2012 y 2016 respectivamente. Para el grupo de escolares, en el género femenino se registró un aumento de $0.4 \%$ en la prevalencia de obesidad, siendo de $11.8 \%$ para el 2012 y de $12.2 \%$ para el 2016 , mientras que en el género masculino también se reportó un aumento de $0.9 \%$, siendo de $17.4 \%$ a $18.3 \%$ para el 2012 y 2016 respectivamente. Finalmente en el grupo de adolescentes tanto en hombres como mujeres se reportó un aumento de la prevalencia de obesidad, en mujeres se registró un aumento de la prevalencia de obesidad de $0.7 \%$, siendo de $12.1 \%$ y $12.8 \%$ en los años 2012 y 2016 respectivamente y en los varones el aumento de la prevalencia fue de $0.5 \%$, de $14.5 \%$ a $15 \%$ en los años 2012 y 2016 respectivamente (Figura 1) 7 .

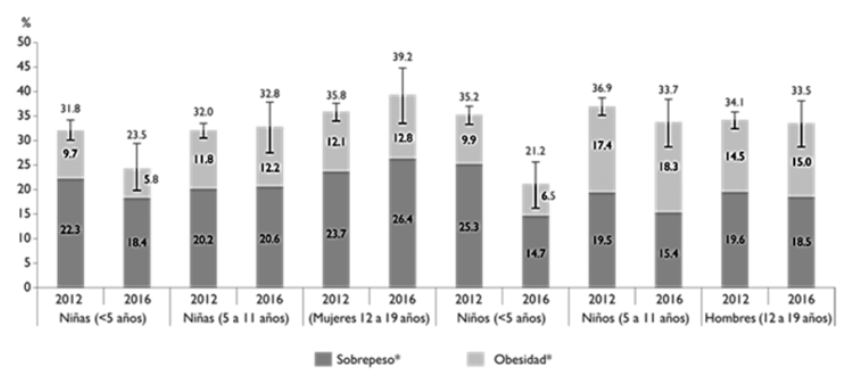

Figura 1. Prevalencia nacional de riesgo de sobrepeso y sobrepeso más obesidad en preescolares ( $<5$ años) y sobrepeso y obesidad en escolares (5 a 11 años) y adolescentes (12 a 19 años), por sexo, en 2012 y 2016. México, ENSANUT MC 2016. 7.

De acuerdo a las cifras mencionadas, México lidera el primer puesto como el país con mayor obesidad infantil del mundo; es decir, muchos niños mexicanos interactúan diariamente con esta enfermedad que en el algún momento culminará en enfermedades como diabetes, hipertensión arterial, cardiopatías, insuficiencia renal, entre otras. A lo largo del tiempo la calidad de vida de la población se ha visto afectada tanto por la modificación de los alimentos, como por las repercusiones ambientales que estas traen consigo, si los alimentos que la población consume se han visto alterados por la mano del hombre es inevitable no considerar las apariciones de diversas complicaciones a futuro y por ende restar años de vida saludable.

\section{Factores que inducen la obesidad}

La obesidad es una condición compleja asociada a muchos factores de riesgo. Parece estar asociada a varios factores, como polimorfismos genéticos, disfunción de la señalización hormonal hipotalámica relacionada con la saciedad, el apetito y el hambre, mayor liberación de adipocinas pro inflamatorias por el tejido adiposo blanco que excede el requerimiento diario de calorías 8 .

Así mismo se asocia con problemas psicosociales, tales como deficiencias en la convivencia social y la calidad de vida. Se ha señalado que los niños obesos tienden a presentar problemas afectivos, principalmente autoestima baja y depresión, por lo cual su imagen 
corporal es negativa y el rendimiento académico e interacción social se ven afectados por los síntomas depresivos y ansiosos, que indicen negativamente en su crecimiento integral 8 .

Herencia genética. En estudios de investigación se encontró que en niños mexicanos existe la presencia de SNP (single nucleotide polymorphism) en genes como MC4R, FTO y ADRB1 asociados a la obesidad, así como también que el polimorfismo PON1-192 que incrementan el riesgo de presentar resistencia a la insulina 9.

Mercadotecnia. Es un factor importante, ya que la exposición a la publicidad de los alimentos ultra procesados hechos a partir de ingredientes industriales, con poco o ningún valor nutricional y con altas cantidad de azúcares, grasas y sodio, la mercadotecnia no sólo influye en la elección de alimentos desde la infancia, sino que también retiene a los consumidores desde una edad temprana 9 .

Sedentarismo. Hoy en día los niños pasan la mayor parte de su tiempo libre frente a los televisores o cualquier dispositivo electrónico como computadora, tabletas electrónicas o teléfonos celulares, lo cual se traduce en inmovilismo que favorece a la obesidad. En épocas pasadas, los juegos requerían mayor cantidad de actividad física; sin embargo, al día de hoy se ven arrasados totalmente por las nuevas tecnologías 9 .

Agentes químicos. Estos actúan como disruptores endocrinos y obesógenos; los primeros son compuestos que interfieren con la producción, liberación, transporte, metabolismo, unión, acción o eliminación de las hormonas naturales responsables del mantenimiento de la homeostasis y de la regulación de los procesos de desarrollo 10 .

Los obesógenos son agentes químicos que pueden promover la obesidad al incrementar el número de células grasas y la acumulaciòn de grasa en estas, al modificar la cantidad de calorías consumidas, alterar el equilibrio energético en favor del almacenamiento de calorías y al modificar los mecanismos mediante los cuales el organismo regula el apetito y la saciedad 9,11 . La hipótesis de los obesógenos ambientales, propone que un subgrupo de disruptores endocrinos (Endocrine disrupting chemicals) pueden promover el desarrollo de la obesidad. Aunque inicialmente controvertida, la hipótesis obesógeno ha ganado adeptos en los años recientes, al identificarse agentes químicos que promueven la adipogénesis y obesidad en animales y humanos 9 .

Entre los obesógenos que pueden afectar al sobrepeso, se encuentran los llamados compuestos orgánicos persistentes (COP), sustancias químicas con elevada resistencia a la degradación, que se acumulan en la cadena alimenticia y que al ser liposolubles, se concentran en las grasas. Estos se encuentran presentes en el medio ambiente y la vida cotidiana, asociados principalmente a insecticidas, pesticidas organoclorados, como el DDT que todavía se detecta en el $88 \%$ de la población a pesar de que se prohibió su uso en 1975 y en menor proporción a perfumes, plásticos o cosméticos 9, 11. A ellos se suman los compuestos denominados seudopersistentes, que aunque no tienen la misma capacidad de fijarse en el organismo, la exposición a estas sustancias es tan continua que también actúan como disruptores endocrinos a diario $g$.

\section{Complicaciones de la Obesidad}

La obesidad está regulada por una serie de adipocinas, entre las cuales se incluyen la leptina, la adiponectina, la haptoglobina y el amiloide sérico; estos mediadores producidos en el tejido adiposo inducen un estado de inflamación sistémica de bajo grado y este proceso es un mecanismo que relaciona la obesidad con un espectro de condiciones patológicas asociadas ${ }_{12}$. De acuerdo al contexto anterior, hablar del síndrome metabólico es conjuntar una variedad de síntomas y signos que incluyen poliuria, polidipsia, astenia, obesidad central, resistencia a la insulina, presión arterial elevada y niveles elevados de glucosa, triglicéridos y colesterol (LDL). De la obesidad se puede derivar este síndrome el cual es muy complejo, ya que crece exponencialmente, por lo tanto, se ha demostrado que la adherencia a un patrón de dieta saludable está inversamente asociada con el síndrome metabólico 13 .

\section{Comorbilidades de la Obesidad Infantil}

La obesidad infantil se asocia con comorbilidades que afectan a casi todos los sistemas del cuerpo, incluidos, entre otros, el sistema endócrino, gastrointestinal, pulmonar, cardiovascular y musculo esquelético. Muchas comorbilidades encontradas en los jóvenes con obesidad, incluyendo diabetes tipo 2, dislipidemia, apnea obstructiva del sueño y esteatohepatitis, las cuales se consideraban como enfermedades de adultos. La severidad de estas comorbilidades es directamente proporcional al grado de obesidad 14 .

Resistencia a la insulina y diabetes mellitus tipo 2

La resistencia a la insulina es una respuesta de glucosa subnormal a la insulina endógena y/o exógena y ocurre más comúnmente en asociación con la obesidad y como consecuencia la resistencia a la insulina relacionada con la obesidad incluyen: intolerancia a la glucosa en ayuno, diabetes mellitus tipo 2 , enfermedad arterial coronaria, síndrome metabólico, síndrome de ovario poliquísitico, enfermedad de hígado graso no alcohólico y ciertas neoplasias malignas relacionadas con la obesidad como cáncer de endometrio ${ }_{15}$.

La resistencia a la insulina tiene un papel importante en el desarrollo de la DM2, reflejado el deterioro de la 


\begin{tabular}{ll} 
Cardiovascular & Neurológico \\
Enfermedad cardiovascular aterosclerótica & Enfermedad vascular cerebral \\
Dislipidemia & Hipertensión intracraneal idiopática \\
Hipertensión & Demencia \\
Insuficiencia cardíaca congestiva & Trastornos musculo esqueléticos \\
Insuficiencia venosa & Osteoartrosis \\
TVP / embolia pulmonar & Limitación de la movilidad \\
Pulmonar & Lumbalgia \\
Apnea del sueño & Genitourinario \\
Síndrome de hipoventilación & Síndrome de ovario poliquístico \\
Asma & Alteraciones de la menstruación \\
Hipertensión pulmonar & Esterilidad \\
Disnea & Incontinencia urinaria de esfuerzo \\
Psicológico & Enfermedad renal terminal \\
Depresión & Hipogonadismo / Impotencia \\
Baja autoestima & Glomerulopatía \\
Inadecuada calidad de vida & Cáncer \\
Trastornos de la alimentación & Metabólico \\
Gastrointestinal & Diabetes tipo 2 \\
Colelitiasis & Intolerancia a la glucosa \\
Enfermedad por reflujo gastroesofágico & Hiperuricemia / gota \\
Enfermedad de hígado graso no alcohólico & Resistencia a la insulina \\
Hernias & Síndrome metabólico \\
Dermatológico & Deficiencia de vitamina D \\
Acantosis nigricans & Cáncer \\
Estrías de distensión & Mama \\
Hirsutismo & Colon \\
Estasis venosa & Próstata \\
Celulitis & Uterino \\
Intertrigo & ----- \\
\hline
\end{tabular}

Cuadro 1. Comorbilidad y complicaciones de la obesidad ${ }_{18}$.

función de las células beta pancreáticas. La severidad de la obesidad sumada a la historia familiar positiva para DM II, son factores importantes para la aparición de este padecimiento ${ }_{16}$.

Apnea obstructiva del sueño

La obesidad es un claro factor de riesgo para que los niños desarrollen síndrome de apnea obstructiva del sueño (SAOS) ${ }_{16}$. Los niños con obesidad tiene $4-6$ veces más de posibilidades de tener SAOS que los niños sin obesidad, se puede sospechar cuando hay somnolencia diurna, ronquidos y episodios de apnea durante el sueño ${ }_{17}$. Este síndrome se ve acompañado por alteraciones en la calidad de vida, el comportamiento, el patrón de crecimiento o los parámetros cardiovasculares y sobre el estado neurocognitivo de los niños ${ }_{16}$.

Dislipidemia
Los niños con obesidad también tienen predominio de otros factores de riesgo incluyendo un perfil de

lipoproteínas caracterizado por aumento de triglicéridos, colesterol total y colesterol de baja densidad (LDL) y niveles bajos de colesterol de alta densidad (HDL). La dislipidemia pediátrica contribuye a la aterosclerosis temprana y por extrapolación a enfermedad cerebrovascular prematura ${ }_{16}$.

Enfermedad de hígado graso no alcohólico

La prevalencia de enfermedad de hígado graso no alcohólico en niños con obesidad varía entre un 11 y $77 \%$. La presentación más leve es la esteatosis caracterizada por acumulación de triglicéridos en los hepatocitos y la forma más avanzada de este problema es la esteatohepatitis en la cual hay daño de las células hepáticas que puede progresar a fibrosis y cirrosis. Este padecimiento se caracteriza por aumento de las 
transaminasas, hiperinsulinismo, disminución de HDL, hipertrigliceridemia y presencia de estrés oxidativo ${ }_{17}$.

\section{MECANISMOS ENDÓGENOS Y EXÓGENOS PARA LA PREVENCIÓN DE LA OBESIDAD.}

Dentro de los mecanismos endógenos tenemos como representantes importantes a los antioxidantes los cuales se pueden dividir en endógenos y exógenos como se muestra en la tabla 1; por otro lado, los mecanismos exógenos para la prevención de la obesidad se pueden dividir en mecanismos de prevención directa e indirecta como se muestran en la tabla 2.

\section{Mecanismos endógenos}

La mayor parte de las enfermedades que padece el ser humano en la actualidad tiene como base una alimentación incorrecta, rica en grasas saturadas, baja en fibra, pobre en vitaminas, entre otras tendencias. En todo caso, los tejidos disponen de una serie de sistemas de defensa para protegerse de una excesiva producción de los radicales libres: los antioxidantes.

Algunos antioxidantes son enzimas y proteínas de alto peso molecular, mientras que otros son pequeñas vitaminas y compuestos moleculares 19 , los cuales poseen magníficas cualidades como la protección celular y la prevención del sobrepeso y la obesidad ya que actúan como antiinflamatorios ${ }_{20}$. Zorrilla, A (2002) señala que la longevidad parece aumentar en concordancia con los niveles de antioxidantes en la dieta y con una reducción calórica; lo que puede propiciar una menor degradación de las mitocondrias, del metabolismo celular y del consumo de oxígeno ${ }_{21}$, a capacidad antioxidante total de los alimentos para eliminar oxidantes está dada por la suma de los productos de la concentración y la constante de velocidad para eliminar el oxidante de cada componente antioxidante 19 . Sus propiedades no sólo deben estudiarse por sus interacciones químicobiológicas, sino por su función en el deterioro oxidativo que los afecta 22 .

Los antioxidantes son compuestos que reducen 0 previenen el estrés oxidativo ya que impiden la formación de óxidos, con lo cual se contrarresta el desequilibrio del estado de oxidación - reducción celular en el que los factores oxidativos netos exceden los factores de reducción netos 23. Cómo funcionan realmente los antioxidantes nutricionales: tono nucleofílico y parahormesis versus eliminación de radicales libres in vivo.

Estos desempeñan sus respectivas funciones en las líneas de defensa al suprimir la producción y eliminación de los oxidantes reactivos reparando así el daño oxidativo; de no ser así se acumularían, de esta manera provocarían estrés oxidativo y un consiguiente

\section{Tabla 1. \\ Antioxidantes endógenos \\ Antioxidante \\ Superóxido dismutasa (SOD)}

Catalasa (CAT)

Glutatión peroxidasa (GPx)

DT-diaforasa

(NADPHquinona oxidoreductasa)

\section{Función}

Enzima encargada de convertir el radical superóxido en una molécula menos reactiva, como el $\mathrm{H}_{2} \mathrm{O}_{2}$, se encuentra en el citosol, interior de mitocondrias y asociada a la matriz extracelular. Utilizado en procesos inflamatorios, daño posisquémico a tejidos y algunos tipos de cáncer 25 .

Se encuentra en mayor medida en los Peroxisomas, y protege la célula de la acumulación de $\mathrm{H}_{2} \mathrm{O}_{2}$ degradándolo hasta convertirlo en agua. Utilizado para tratar el daño por inflamación y en la enfermedad xerodermica pigmentosa ${ }_{25}$.

Enzima selenio (Se) dependiente que cataliza la reducción del peróxido de hidrógeno $\left(\mathrm{H}_{2} \mathrm{O}_{2}\right)$ o lipoperóxido $(\mathrm{L}-\mathrm{OOH})$, utilizando como agente reductor el glutatión reducido $(\mathrm{GSH}) 26$.

Enzima antioxidante que cataliza la reducción de diversas quinonas hasta sus correspondientes hidroquinonas. Esencial en su papel como antioxidante y en la citoprotección frente a sustancias tóxicas, ya que evita la generación de intermediarios semiquinónicos, los cuales presentan una elevada tendencia a reaccionar con el oxígeno dando lugar a superóxido 27 .

Hormona secretada por la glándula pineal. Estudios hechos en ratas confirman que dosis bajas de melatonina favorecen el tránsito intestinal y el vaciado gástrico, 
mientras que dosis altas lo inhiben. Antioxidante, a través de la activación de las enzimas antioxidantes glutatión peroxidasa, glutatión reductasa, glucosa-6fosfatodeshidrogenasa, catalasa y superóxido dismutasa, las vitaminas $\mathrm{E}$ y $\mathrm{C}$ y el aumento de la cadena respiratoria 28 .

\section{Antioxidantes exógenos Antioxidante Vitamina C}

\section{Carotenoides}

Vitamina E ( $\alpha$-tocoferol)

Flavonoides

\section{Función}

Se encuentra extra e intracelularmente, es capaz de reaccionar directamente con el radical superoxido, radical hidroxilo e hidroperóxidos lipídicos. Además, restaura las propiedades antioxidantes de la Vitamina $\mathrm{E}_{29}$.

Fuentes: Guisantes, papas, nabos, tomates, naranja, limón y col cruda 25 .

Precursor de la Vitamina A. Secuestra oxígenos singlete, es capaz de reaccionar con los radicales peroxilo, inhibe la propagación de la lipoperoxidación de las membranas 29 . Fuentes: Zanahoria, tomate, camote, duraznos, maíz amarillo, entre otras hortalizas y frutas amarillas 25 .

De todos los tocoferoles, el $\alpha$-tocoferol es el que posee mayor actividad antioxidante. Es de carácter lipofílico, capaz de reaccionar con especies reactivas de oxígeno, convirtiéndolos en hidroperóxidos lipídicos 29 .

Fuentes: Leche, huevo, carne de res y pescado, semillas de trigo y otra garmíneas 25 .

Tienen propiedades anti-radicales libres que se dirigen fundamentalmente hacia los radicales hidroxilo y superóxido, capacidad de modificar la síntesis de eicosanoides (con respuestas anti-prostanoide y anti-inflamatoria), de prevenir la agregación plaquetaria (efectos antitrombóticos) y de proteger a las lipoproteínasde baja densidad de la oxidación (prevención de la placa de ateroma).

Fuentes: cerveza, vino, té verde, té negro, soja, arándano, gingko biloba, cardo, mariano o crataegus 30 .

De forma general podemos sustentar que, una adecuada alimentación o simplemente la nutrición equilibrada cumple dos objetivos primordiales durante la infancia: 1) conseguir un estado nutritivo óptimo, mantener un ritmo de crecimiento adecuado y tener una progresivo desequilibrio entre la producción de especies reactivas de amortiguar rápidamente los reactivos para reparar el daño resultante, por consiguiente, cambiaría la estructura celular, desarrollando deterioro de los tejidos, favoreciendo la aparición de diferentes patologías ${ }_{20}$. Un estudio reveló que el estrés oxidativo parece estar significativamente involucrado en el desarrollo de complicaciones relacionadas con la obesidad 24 .

De acuerdo al planteamiento anterior, existen dos vías fundamentales para proteger al organismo de los radicales libres; enzimáticos (endógenos) y no enzimáticos (exógenos) y su interacción puede ser intracelular o extracelular. La vía endógena requiere apoyo externo y por ello se recomiendan los antioxidantes exógenos cuyo papel es relevante dado que son los que pueden formar parte de la dieta diaria 22 madurez biopsicosocial y 2) establecer recomendaciones dietéticas que permitan prevenir enfermedades de origen nutricional que se manifiestan en la edad adulta pero que inician durante la infancia ${ }_{31}$.

La alimentación durante la infancia y preadolescencia tiene una gran trascendencia en la proyección de la calidad de vida del adulto, un ejemplo es la dieta mediterránea, basada en un bajo

\section{Mecanismos Exógenos}

Introducción tardía a la ablactación.

Un estudio australiano de seguimiento en 620 sujetos nacidos entre 1990 y 1994 encontró que la introducción tardía de la alimentación complementaria (6 meses o más) se asoció con menor riesgo de sobrepeso y obesidad a los 10 años 32 .

Edulcorantes adicionales

El añadir sal y azúcar en la preparación de los alimentos para lactantes durante el primer año de vida es innecesario, pues ambos componentes se encuentran en 
cantidades suficientes de forma natural en la dieta refuerzan la preferencia innata por los sabores, por lo tanto, los edulcorantes suponen un riesgo añadido de caries dentales y obesidad 32 .

Ingesta de bebidas carbonatadas

Las bebidas carbonatadas como los jugos artificiales y gaseosas no se recomiendan, debido a que disminuyen la absorción de calcio, proveen altas cantidades de energía, carecen de valor nutrimental, disminuyen el apetito y la aceptación de otros nutrientes. Es conveniente evitar estas bebidas con aparente valor nutricional lo cual no es cierto, debido a que únicamente es una costumbre cultural, promoviendo la obesidad ${ }_{33}$.

\section{Lactancia materna}

Los niños y niñas que habían sido amamantados durante al menos 6 meses tuvieron de un 22 a 30\% menos probabilidades de sobrepeso y más de un $40 \%$ menos probabilidades de obesidad 6 .

La relación entre la lactancia materna exclusiva y el menor riesgo de desarrollo de obesidad, como la introducción más tardía de la alimentación complementaria y la menor ingesta energética y proteica, podrían estar mediados por una menor liberación de insulina $y / u$ otras hormonas pancreáticas e intestinales en los lactantes amamantados o por el contenido de la leche materna en hormonas y otros factores biológicos, así como la distinta respuesta a los mecanismos de hambre y saciedad cuando se comparan con lactantes alimentados con una fórmula infantil 34 .

\section{Limitar el tamaño de las raciones}

La OMS señala que los resultados de un número limitado de estudios indican la existencia de una relación entre la disponibilidad de raciones más grandes y un incremento de la ingesta calórica total, lo que podría llevar a un aumento de peso. Por tanto, limitar el tamaño de las raciones puede ser una intervención eficaz para evitar comer en exceso y posiblemente reducir el riesgo de sobrepeso y obesidad entre los niños ${ }_{35}$. Incrementar consumo de frutas y verduras

Un bajo consumo de frutas y verduras está asociado a una mala salud y a un mayor riesgo de enfermedades no transmisibles ${ }_{36}$. Por su alto contenido de micronutrientes (vitaminas, minerales y fibra) tienen los siguientes beneficios: Reducen los niveles de colesterol, mejoran el tránsito intestinal, disminuyen los niveles de glucosa en sangre, contribuyen a la eliminación de toxinas, aumentan de la sensación de saciedad evitando el consumo exagerado de alimentos que conducen al sobrepeso y la obesidad ${ }_{37}$. Se estima que en 2017 unos
3.9 millones de muertes se debieron a un consumo inadecuado de frutas y verduras 36 .

Disminuir las horas de Televisión

Hay dos consecuencias de comer y ver televisión, por una parte propicia la vida sedentaria y por otro el niño recibe información de alimentos de bajo contenido nutricional y de alto contenido calórico, lo cual conlleva al sobrepeso y a la obesidad. La American Academy of Pediatrics refiere que los niños que ven televisión más de cuatro horas por día tenían un Índice de Masa Corporal (IMC) mayor comparado con el de los niños que ven televisión menos de dos horas por día. Así como el tener un televisor en la recamara es un fuerte pronosticador de tener sobrepeso, aún en los niños preescolares 38, 39, 40. Promover estilo de vida activo

Para llevar una vida sana, es importante ofrecer a los niños actividades físicas atrayentes y orientar a sus cuidadores de cómo seguir las recomendaciones sobre estas actividades, así como el número adecuado de horas que los niños deben dormir o ver la televisión 41,42 .

El aumento de las oportunidades de participar en actividades físicas estructuradas y no estructuradas que sean seguras, apropiadas e idóneas para personas de uno u otro sexo, tanto dentro como fuera de la escuela, incluido el transporte activo (desplazamiento a pie y en bicicleta), tendrá efectos beneficiosos y colaterales que repercutirán en la salud, el comportamiento y el nivel educativo de todos los niños y adolescentes. La OMS informa que el $81 \%$ de los adolescentes no alcanzan los 60 minutos diarios de actividad física recomendada 43 .

\section{Detrimento de la autoestima}

Actualmente nuestra sociedad tiene una evidente aversión a la gordura, se considera ideal el prototipo de figura esbelta y delgada relacionándola con triunfo y valía personal, esto pone a las personas obesas en una posición difícil, sobre todo a las adolescentes femeninas que padecen obesidad, para quienes esta presión es más evidente. Podríamos pensar que lo antes mencionado es a causa de presiones externas, pero sin duda, se trata de una experiencia íntima sobre lo que el individuo siente y piensa de sí mismo y no sobre lo que otras personas opinan de él 44 , sintiendo vergüenza sobre su cuerpo y falta de confianza en sí mismo, esto podría ocasionar un rendimiento académico deficiente en la escuela, ausentismo escolar y retraimiento social, por mencionar algunas de sus consecuencias. 
Tabla 2. Mecanismos exógenos de prevención directa e indirecta.

\begin{tabular}{ll}
\hline \multicolumn{1}{c}{ Prevención Directa } & Introducción tardía a la ablactación \\
Edulcorantes adicionales & Lactancia materna \\
Disminuir las horas de televisión & Promover estilo de vida activo \\
Ingesta de bebidas carbonatadas & Orientar a la población sobre el control de tamaños de porción \\
Incrementar el consumo diario de frutas y verduras & Prendiran
\end{tabular}

Un estudio desarrollado por Sánchez Arenas et al (2015) confirmó que existe relación entre autoestima e imagen corporal en niños mexicanos con obesidad. La mayoría de los niños participantes presentaron autoestima baja, tanto en su evaluación total como en áreas personales como: yo, familia, fracaso, éxito, intelectualidad y afectivo-emocional, y se determinó que presentan insatisfacción con su imagen corporal, ya que se identifican con figuras más delgadas en comparación con su complexión real 44 .

\section{CONCLUSIÓN}

México, al estar en el primer puesto a nivel mundial en obesidad infantil, tiene un asunto urgente que resolver. La sociedad necesita ser impulsada con disciplina en acciones y programas promovidos por el gobierno para desmantelar conductas de riesgo a la salud. La Organización Mundial de la Salud (OMS) advierte que una de las principales acciones para evitar la obesidad, es impulsar políticas destinadas a generar actividades físicas y deporte, reafirmando la misma intención la Organización Panamericana de la Salud (PAHO), donde también incluye el mejoramiento de los ambientes escolares de alimentación, la promoción de la lactancia materna y la alimentación saludable. Al impulsar estas actividades benéficas, el costo que representa la atención médica de las enfermedades atribuibles al sobrepeso y la obesidad debe disminuir considerablemente, tanto para el gobierno como para la propia economía familiar. Todas estas estrategias para la reducción de la obesidad y con ello las complicaciones que esta epidemia conlleva, confluyen indiscutiblemente en la aplicación de una disciplina médica, la Medicina Preventiva, que, si bien no es innovadora, ha sido subestimada en la práctica debido a la falta de promoción de la salud, así como a su mantenimiento y el bienestar en los grupos donde mayor oportunidad existe, la infancia y adolescencia. Por lo tanto, la principal conclusión de este trabajo es llevar a cabo las acciones planteadas para prevenir la obesidad infantil y con esto seguramente futuros adolescentes obesos y adultos con enfermedades crónicodegenerativas, son casi ilimitadas. Por último, sin minimizar su importancia, se ha unido la investigación, que sin duda ha otorgado el impulso determinante a la generación de políticas de salud pública. Al momento, esta investigación nos arroja los vastos mecanismos de protección y prevención de las complicaciones de la obesidad en los adolescentes, quedando a la expectativa de los resultados de la aplicación de la oportuna Medicina Preventiva antes planteada.

\section{Referencias}

[1] Organización Mundial de la Salud. Estrategia mundial sobre régimen alimentario, actividad física y salud, ¿Qué son el sobrepeso y la obesidad? Recuperado

https://www.who.int/dietphysicalactivity/childhood_what/es/

[2] Marino Rosa FM, et al. Respuestas cardiovasculares de mujeres con obesidad mórbida sometidas a un test ergoespirométrico con ergómetro de brazo. Revista colombiana de Cardiología. 2017; 24(5): 532-536.

[3] Senado de la República. Coordinación de comunicación social. México ocupa el primer lugar a nivel mundial en Obesidad infantil y el segundo en adultos: UNICEF. Recuperado de: http://comunicacion.senado.gob.mx/index.php/informacion/boletines/87internacional/39127-mexico-ocupa-el-primer-lugar-a-nivel-mundial-enobesidad-infantil-y-el-segundo-en-adultos-unicef.html

[4] UNICEF. Informe Annual. México 2017. Recuperado de: https://www.unicef.org.mx/Informe2017/Informe-Anual-2017.pdf

[5] Pérez-Herrera A y Cruz M. Situación actual de la obesidad infantil en México. Nutr Hosp. 2019; 36(2): 463-469.

[6] Córdova Villalobos JA, et al. Sobrepeso y obesidad. Situación actual y perspectivas. Acta Médica Grupo Ángeles. 2010; 8(4): 202-207.

[7] Shamah-Levy TT, et al. Sobrepeso y obesidad en niños y adolescentes en México, actualización de la Encuesta Nacional de Salud y Nutrición de Medio Camino 2016. Salud Pública de México. 2018; 60(3): 244-253.

[8] Delgado-Floody P, et al. Relación entre sobrepeso, obesidad y niveles de autoestima en escolares. Retos. 2019; 35: 67-70.

[9] Doadrio V AL. Role of obesogens in adipogenicity and obesity. An Real Acad Farm. 2016; 82: 206-214.

[10] Kavlock R J, et al. Research needs for the risk assessment of health and environmental effects of endocrine disruptors: a report of the U.S. EPA-sponsored workshop. Environ Health Perspect. 1996; 104(4): 715740.

[11] Macías M AI, et al. Hábitos alimentarios de niños en edad escolar y el papel de la educación para la salud. Revista Chilena de Nutrición. 2012; 39(3): 40-43.

[12] Singer K y Lumeng CN. The initiation of metabolic inflammation in childhood obesity. J Clin Invest. 2017; 127(1): 65-73. 
[13] Calder PC, et al. Dietary factors and low-grade inflammation in relation to overweight and obesity. British Journal of Nutrition. 2011; 106(S3): S5-S78.

[14] Kumar S \& Kelly AS. Review of childhood obesity: from epidemiology, etiology, and comorbidities to clinical assessment and treatment. Mayo Clin. Proc. 2017; 92(2): 251-265.

[15] Semple RK et al. Síndromes genéticos de resistencia severa a la insulina. Endrocr Rev. 2011; 32: 498.

[16] Alonso A M.L, et al. Tratamiento del síndrome de apnea obstructiva del sueño en niños: más opciones, más confusión. Arch Bronconeumol. 2018; 54(8): 409-411.

[17] Raimann TX. Obesidad y sus complicaciones. Rev. Med. Clin. Condes. 2011; 22(1): 20-26.

[18] Catenacci VA, et al. The obesity epidemic. Clinics in Chest Medicine. 2009; 30(3): 415-444.

[19] Valdecantos MP, et al. Obesidad y estrés oxidante: papel de la suplementación con antioxidantes de la dieta. Revista de Investigación Clínica. 2009; 61(2): 127-139.

[20] Huberman A. La importancia médica de los radicales libres de oxígeno. Gac Méd Méx. 1996; 132(2): 184-195.

[21] Zorrilla A. El envejecimiento y el estrés oxidativo. Rev. Cubana Invest. Biomed. 2002; 21(3): 178-185.

[22] Coronado $\mathrm{H} \mathrm{M}$, et al. Antioxidantes: perspectiva actual para la salud humana. Rev Chil Nutr. 2015; 42(2): 206-212.

[23] Forman $\mathrm{H} \mathrm{J}$ et al. Cómo funcionan realmente los antioxidantes nutricionales: tono nucleofílico y parahormesis versus eliminación de radicales libres in vivo. Radic Free Biol Med. 2014; 66: 24.

[24] Hutcheson R y Rocic P. The metabolic syndrome, oxidative stress, environment, and cardiovascular disease: The great exploration. Experimental Diabetes Research. 2012; 2012: 1-3.

[25] Laguna, 2013. Bioquímica de Laguna. 7a ed. México, D.F: Manual Moderno. Cap. 24, p. 426 - 428; Cap. 12, p. 178 - 179.

[26] Cisneros Prego E et al. Enzimas que participan como barreras fisiológicas para eliminar los radicales libres: III. Glutatión peroxidasa. Revista Cubana de Investigaciones Biomédicas. 1997; 16(1): 10-15. Recuperado

http://scielo.sld.cu/scielo.php?script=sci_arttext\&pid=S086403001997000100002

[27] Jódar Montilla L. Papel de la NAD (P) H: quinona oxidorreductasa 1 (NQO1) en el control del crecimiento de las células animales. (Tesis Doctoral). Departamento de biología celular, fisiología e inmunología Universidad de Córdoba. 2010.

[28] Pal PK y Maitra SK. Response of gastrointestinal melatonin, antioxidants, and digestive enzymes to altered feeding conditions in carp (Catla catla). Fish Physiology and Biochemistry. 2018; 44(4): 1061-1073.

[29] Gil Hernández A. 2010. Tratado de Nutrición, Tomo 1, Bases Fisiológicas y Bioquímicas de la Nutrición. 2a ed. Madrid: Médica Panamericana, Cap. 18, p. 472 y 475.

[30] Martínez Flórez S et al. Los flavonoides: propiedades y acciones antioxidantes. Nutr. Hosp; 2002; 17(6): 271-278.

[31] Palenzuela Paniagua SM et al. La alimentación en el adolescente. An. Sist. Sanit. Navar. 2014; 37(1): 47-58.
[32] Seach KA et al. Delayed introduction of solid feeding reduces childover weight and obesity at 10 years. International Journal of Obesit. 2010; 34(10): 1475-1479.

[33] Perdomo Giraldi M \& Durán FM. Alimentación complementaria en el lactante. Pediatr Integral. 2015; 19(4): 260-267.

[34] Moreno Villares JM et al. Los primeros 1000 días: una oportunidad para reducir la carga de las enfermedades no transmisibles. Nutr Hosp. 2019; 36(1): 218-232.

[35] Organización Mundial de la Salud, Limitar el tamaño de las raciones para reducir el riesgo de sobrepeso y obesidad infantil 2019. Recuperado de: https://www.who.int/elena/titles/portion_childhood_obesity/es/ [36] Organización Mundial de la Salud, Aumentar el consumo de frutas y verduras para reducir el riesgo de enfermedades no transmisibles, Biblioteca electrónica de documentación científica sobre medidas nutricionales (eLENA) 2019. Recuperado de: https://www.who.int/elena/titles/fruit_vegetables_ncds/es/

[37] ABECÉ. Promoción del consumo de frutas y verduras. MINSALUD. Imprenta Nacional de Colombia. Colombia. [2015]. Recuperado de: https://www.minsalud.gov.co/sites/rid/Lists/BibliotecaDigital/RIDE/VS/P P/ENT/abece-frutas-y-verduras.pdf

[38] Powell LM et al. Nutritional content of television food advertisements seen by children and adolescents in the United States. PEDIATRICS. 2007; 120(3): 576-583.

[39] Reyes Gómez U et al. La televisión y los niños: II Obesidad. Boletín Clínico Hospital Infantil del Estado de Sonora. 2006; 23(1): 25-30.

[40] Krebs NF, Jacobson MS, American Academy of Pediatrics Committee on Nutricion, (2003). Prevention of Pediatric Overweight and Obesity. PEDIATRICS, 112(2):424-430.

[41] LeBlanc AG et al. Systematic review of sedentary behaviour and health indicators in the early years (aged 0-4 years). Appl Physiol Nutr and Metab. 2012; 37(4): 753-772.

[42] Taveras EM et al. Chronic sleep curtailment and adiposity. Pediatrics. 2014; 133(6): 1013-1022.

[43] Organización Mundial de la Salud, INFORME DE LA COMISIÓN PARA ACABAR CON LA OBESIDAD INFANTIL 2016. Recuperado de:

https://apps.who.int/iris/bitstream/handle/10665/206450/9789243510064_ spa.pdf

[44] Sánchez Arenas JJ \& Ruiz Martínez AO. Relationship between selfesteem and body image in children with obesity. Revista Mexicana de Trastornos Alimentarios. 2015; 6(1): 38-44. 